\title{
Integral and separable dimensions of shape
}

\author{
STUART I. OFFENBACH \\ Purdue University, West Lafayette, Indiana
}

\begin{abstract}
The development of holistic and analyzable dimensional processing was studied in school-age children and college adults using different shapes in an unrestricted sorting task. A model is described in which the dimensions of stimulus differences are carefully defined as having either a perceptual base or a conceptual base. Predictions based on the model were that the developmental trend from integral to separable responding would not be observed. The results supported the model. Most children and adults made judgments on the basis of overall similarity. Only secondgrade children showed evidence of increased dimensional analysis. The implication of these results for other approaches to perceptual organization is discussed.
\end{abstract}

Perceptual organization has been studied by examining how children group or match stimuli that are presumed to be either exemplars of a concept or related in some dimensional structure. Garner's proposal $(1974,1983)$ that some combinations of stimulus dimensions interact to produce percepts that are holistic or integral, whereas other dimensional combinations generate compounds that can be analyzed or separated into their component attributes (called separable dimensions), has been extended to account for developmental findings by Shepp and his colleagues (e.g., Kemler, 1983; Shepp, 1978, 1983). On the basis of the results of studies of children's performance on various sorting tasks, Shepp (1978) proposed that young children's perceptual organization is primarily holistic, and that older children's and adults' perceptual organization is based on the ability to analyze multidimensional compounds into component dimensions. Furthermore, some dimensional combinations perceived as separable by the older child and adult are perceived as integral by the younger child.

Integral and separable compounds are processed in different ways. For example, the similarity of two integral compounds is based on their overall appearance and is detected quite rapidly. Separable compounds, on the other hand, are more slowly decomposed into their constituent dimensions, and they are judged to be similar if there is a shared dimensional value. One of the several tasks that have been used to demonstrate these differences is the unrestricted sorting task (cf. Kemler, 1983, and Shepp, 1983, for reviews). In this task, children are shown three compounds, and they must identify which two are most similar. Two of the compounds share a dimensional value (e.g., both are squares, but have different sizes), but the

This research was supported by Grant HD20431 from the National Institute of Child Health and Human Development. Portions of this research were reported at the 1988 meeting of the Psychonomic Society. Thanks are extended to Laura Curry and to the Frankfort, Indiana, Community School Corporation and the Tippecanoe School Corporation for their help in various aspects of this research. Correspondence may be addressed to Stuart I. Offenbach, Department of Psychological Sciences, Purdue University, West Lafayette, IN 47907. third, which differs on both dimensions (a hexagon of intermediate size), would be psychophysically more similar to the larger of the two squares. When the compounds are integral, the hexagon and larger square are perceived as being most similar. Saying the two squares are most similar would reflect a dimensional analysis of separable compounds. Young preschool children would be more likely to group the hexagon and larger square, and older children should state that the two squares are most similar.

One confusing aspect of the developmental separability hypothesis is that a dimension may produce a holistic compound in combination with one other dimension and an analyzable compound when paired with a different dimension. Foard and Kemler Nelson (1984) reported that brightness combined with saturation differences produced integral compounds, and brightness combined with size yielded separable compounds. No theoretical explanation was given to account for these different perceptual organizations.

The research reported here tests a hypothesis proposed by Offenbach and Blumberg (1985) that does make a priori predictions as to when dimensional combinations yield integral and holistic compounds on the one hand and separable, analyzable compounds on the other. The hypothesis is conditioned on two types of stimulus dimensions: perceptual dimensions that are based on physical differences among item exemplars, and conceptual dimensions, which are derived from internally mediated representations of a set of stimuli (often reflecting a representation in memory of earlier perceptual attributes). Variations in shape provide a convenient example of this developmental process. Two perceptual dimensions form the basis of differences in common geometric shapes (Offenbach, 1983, Experiment 1)-the number of sides, and compactness (a dimension based on the ratio of the perimeter to area; cf. Shepard, 1962). Pairs of shapes may differ on just one dimension (e.g., a triangle and a plus sign differ in the numbers of sides) or on both dimensions (e.g., triangles and hexagons each have different values on the sides and the compactness dimensions). With increasing age, these shape variations come to be 
represented in memory as a "unified dimension of shapes," independent of their underlying perceptual dimensions.

Children with no conceptual representation of a stimulus domain can organize compounds only on the basis of perceptual dimensions, a developmentally earlier level of processing. Young children may be capable of analyzing compounds that are generated from the perceptual dimensions of a domain, but not those which are generated from conceptual dimensions (Offenbach \& Blumberg, 1985). Older children and adults, who have a representational structure for simple dimensions such as shape and color, can override the more primitive perceptual dimensions. The result should be that these older children interpret all shape compounds as exemplars of a single unidimensional shape category. The likely basis for sorting such unidimensional compounds would be overall similarity. For shapes generated by combining numbers of sides and compactness, the developmental trend from integral to separable responding should be absent (although children who are just beginning to generate conceptual representations might show more dimensional analysis).

This hypothesis was tested using an unrestricted sorting task. Children and adults were asked to select which two of three shapes "went together best" (or which compound "did not belong with the others"). Two of the shapes shared a value on one dimension (e.g., compactness) and differed considerably on the third dimension (number of sides). The third compound differed on both these perceptual dimensions. The model predicts that the developmental trend from integral selections to analyzable selections should not be observed.

\section{METHOD}

\section{Subjects}

Elementary school children from kindergarten $\left(M_{\text {age }}=75.4\right.$ months; $N=12)$, Grade $2\left(M_{\text {age }}=100.1\right.$ months; $\left.N=18\right)$, Grade $4\left(M_{\text {age }}=\right.$ 124.5 months; $N=15)$, and Grade $6\left(M_{\text {age }}=146.1\right.$ months; $\left.N=9\right)$, and adults from introductory psychology classes at Purdue University served as subjects $\left(M_{\text {age }}=240.1\right.$ months; $\left.N=25\right)$. The children all were from schools in rural areas of Indiana.

\section{Procedure}

Each participant, child or adult, was administered a 32-trial, threechoice unrestricted sorting task (an Amiga 1000 computer was used to generate stimuli and record responses). One shape was located in the top center of the computer monitor, one in the bottom left, and the third in the bottom right. The subjects responded by pressing keys that corresponded to the positions of the three shapes on the screen. Six geometric shapes were used to construct eight stimulus triads. The dimensional coordinates for each of the six compounds may be found in Table 1. Four shapes-isosceles triangle, right triangle, plus sign (cross), and five-point star-had almost identical values on the compactness dimension and very different values on the number-of-sides dimension. The remaining two shapes, a diamond and a hexagon, differed from the others on both the compactness and number-of-sides dimensions.

This study had one variable between groups (the five ages) and one variable within subjects (response required). On half of the trials, the subjects selected the two shapes that "went together best" (inclusion trials), and on the remaining trials, they selected the "shape that did not belong with the others"' (exclusion trials). The children were tested individually, in a room provided by the cooperating school, and the college adults were tested in the Developmental Psychology Research Labs (also individually). Inclusion trials preceded exclusion trials, and each
Table 1

Dimensional Values of Shape Compounds

\begin{tabular}{lcc}
\hline \multicolumn{1}{c}{ Shapes } & No. of Sides & Compactness \\
\hline Right triangle & +.50 & -.20 \\
Isosceles triangle & +.54 & -.22 \\
Star & -.35 & -.31 \\
Plus & -.30 & -.31 \\
Diamond & +.23 & +.20 \\
Hexagon & +.38 & -.12 \\
\hline
\end{tabular}

of the eight triads was seen twice for each type of trial. Practice trials were administered until each participant seemed to understand the task and the response to be made (even the youngest children required only two or three practice trials). The procedure was self-paced, with the subjects initiating each trial by pressing a start key (the subjects were asked to use the same finger to press start and response keys).

\section{RESULTS}

The number of separable, integral, and haphazard responses for the inclusion and exclusion trials was tabulated for each subject (haphazard $=$ responses not predicted by the model). The probabilities of holistic, analyzable, and haphazard responses on inclusion trials and exclusion trials were not significantly different $\left(z_{\text {analyzable }}=1.44\right.$, $z_{\text {holistic }}<1.00$, $z_{\text {haphazard }}=1.66$, all $\left.p s<.05\right)$. Asking children and adults to include stimuli in a matched set or to exclude an "odd" stimulus did not influence their matching judgments.

A summary of the proportion of holistic, analyzable, and haphazard responses for each age level is included in Table 2. A chi-square analysis computed to examine the relationship among types of responses with age level was significant $\left[\chi^{2}(8)=70.79, p<.05\right]$. More than half of the children's responses were classified as holistic, similarity-based responses. The frequency of analyzable responses was very low for all but second-grade children, who showed evidence of increased dimensional responding.

\section{DISCUSSION}

The results support the prediction that compounds varied on perceptual dimensions would be organized on the basis of overall similarity. The majority of the children and adults matched shapes on the basis of overall similarity rather than dimensional analysis and common features. There was no discernible tendency for older children or college adults to make more analyzable matches. Older children and adults apparently viewed the compounds as exemplars of a single shape dimension. Because the concept of integral and analyzable compounds is not applicable to unidimensional stimuli, those sorts are based on overall similarity. Such responses only resemble true integral or holistic responses (which require multidimensional compounds). The increase in the percentage of such integral responses among the older children

Table 2

Response Probabilities

\begin{tabular}{lccc}
\hline \multicolumn{1}{c}{ Grade } & Integral & Separable & Haphazard \\
\hline Kindergarten & .497 & .138 & .424 \\
Grade 2 & .450 & .172 & .358 \\
Grade 4 & .469 & .073 & .458 \\
Grade 6 & .479 & .045 & .476 \\
College & .540 & .034 & .426 \\
\hline
\end{tabular}


and adults occurred because of the developmental changes in the conceptual understanding of shape.

These results are also consistent with the position taken by Ward and his colleagues (Ward, Foley, \& Cole, 1986; Ward \& Vela, 1986). Ward noted that other factors may influence perceptual organization (e.g., experience with a dimension and individual differences in cognitive tempo are two such factors ${ }^{1}$ ). Thus, Ward and Vela (1986) reported that adults were more holistic than children when processing very familiar color compounds. Ward proposed that experience with a stimulus domain (color in this case) enabled the adults to integrate the separate color dimensions (hue, saturation, and brightness) into "higher-order wholes" or what I would call a unidimensional conceptual dimension. Children who lacked extensive experience with colors analyzed the compounds into component dimensions and then matched compounds by common values. The age differences here were similar to Ward's-the youngest children responded to the perceptual dimensions and matched on the basis of overall perceptual similarity, the second graders probably had a conceptual representation of color but lacked experience and made more separable responses (perhaps revealing a transitional period between holistic perception and unidimensional organization), and the older children and adults viewed the stimulus variations as unidimensional. These results support the premise that when and how children and adults analyze stimulus compounds into component dimensions is a more complex process than originally thought. While the underlying structure of the dimensions (perceptual or conceptual) influences categorization, the limits of that influence must be identified in order to have a more complete understanding of conceptual development.

\section{REFERENCES}

FoARD, C. F., \& Kemler Nelson, D. G. (1984). Holistic and analytic modes of processing: The multiple determinants of perceptual analysis. Journal of Experimental Psychology: General, 113, 94-111.

GARNER, W. R. (1974). The processing of information and structure. Potomac, MD: Erlbaum.

GARNER, W. R. (1983). Asymmetric interactions of stimulus dimensions in perceptual information processing. In T. J. Tighe \& B. E. Shepp (Eds.), Perception, cognition, and development: Interactional analyses (pp. 2-38). Hillsdale, NJ: Erlbaum.
KemLer, D. G. (1983). Holistic and analytic modes in perceptual and cognitive development. In T. J. Tighe \& B. E. Shepp (Eds.), Perception, cognition, and development: Interactional analyses (pp. 77102). Hillsdale, NJ: Erlbaum.

OFFENBACH, S. I. (1983). The concept of dimension in research on children's learning. Monographs of the Society for Research in Child Development, 48(6, Serial No. 204).

OfFENBACH, S. I., \& Blumberg, F. C. (1985). The concept of dimensions in developmental research. In H. W. Reese (Ed.), Advances in Child Development and Behavior (Vol. 19, pp. 85-115). Orlando, FL: Academic Press.

SHEPARD, R. N. (1962). The analysis of proximities: Multidimensional scaling with an unknown distance function: II. Psychometrika, 27, 219-246.

SHEPP, B. E. (1978). From perceived similarity to dimensional structure: A new hypothesis about perceptual development. In E. Rosch \& B. B. Lloyd (Eds.), Cognition and categorization (pp. 135-167). Hillsdale, NJ: Erlbaum.

SHEPP, B. E. (1983). The analyzability of multidimensional objects: Some constraints on perceived structure, the development of perceived structure, and attention. In T. J. Tighe \& B. E. Shepp (Eds.), Perception, cognition, and development: Interactional analyses (pp. 39-75). Hillsdale, NJ: Erlbaum.

WARD, T. B., FolEY, C. M., \& CoLE, J. (1986). Classifying multidimensional stimuli: Stimulus, task, and observer factors. Journal of Experimental Psychology: Human Perception \& Performance, 12, 211-225.

WARD, T. B., \& VELA, E. (1986). Classifying color materials: Children are less holistic than adults. Journal of Experimental Child Psychology, 42, 273-302.

\section{NOTE}

1. Ward qualified his results, indicating the cognitive tempo relationship might hold for some dimensional combinations (e.g., size and brightness) and not for others (e.g., saturation and brightness).

(Manuscript received May 19, 1989.) 